\title{
Dorothea Kübler
}

\section{Marktdesign und die Verteilung von Impfstoff}

Gütermärkte sind einfach: Wenn man bezahlen kann, bekommt man, was man will. Aber viele wichtige Güter kann man nicht einfach auswählen, sondern muss auch ausgewählt werden. Eine Schule kann man sich zwar aussuchen, aber man muss auch einen Platz bekommen. Auch bei Studienplätzen wählt bei knappen Plätzen die Uni aus, wer zugelassen wird. Spendernieren kann man nicht kaufen, sondern man muss nach der Warteliste an der Reihe sein bzw. einen passenden Spender haben. Und Lebenspartner wählen sich gegenseitig. Auch Termine für öffentliche Dienstleistungen, etwa Passangelegenheiten, Visa etc. kann man nicht kaufen, sondern muss an der Reihe sein. Das hat früher bedeutet, dass man lange Wartezeiten auf sich nehmen musste, während man heute meistens einen Termin mit Hilfe von Online-Terminvergabesystemen bucht. Ähnliches gilt für die Verteilung von Impfstoff in der Pandemie, solange der Impfstoff knapp ist. Fast überall auf der Welt wird priorisiert, wer geimpft werden kann, und es werden Termine an priorisierte Gruppen vergeben.

Warum spielt Geld für diese Güter keine Rolle? Es gibt andere Kriterien, die darüber entscheiden, wer was bekommt. Die eigenen Leistungen können ausschlaggebend sein, etwa für Plätze an Schulen und Universitäten. Bei Schulen zählen häufig auch soziale Kriterien, denn Schulen haben eine gesellschaftliche Integrationsfunktion, und Bildung entscheidet über soziale Mobilität. Oft ist es auch verboten, Geld zu verwenden, etwa für menschliche Organe. Bei Impfterminen oder Terminen bei öffentlichen Ämtern sollen alle Menschen den gleichen Zugang haben, unabhängig von ihrem Einkommen. Zusätzlich gilt, dass bei Impfungen die Impfpriorisierung, also das Alter und die Anfälligkeit für Krankheiten oder schwere Verläufe, berücksichtigt werden soll.

„Lokale Gerechtigkeit“ bezüglich zentraler Güter wie etwa Gesundheit und Bildung muss von eigens dafür eingesetzten Institutionen sichergestellt werden (Elster, 1992). Lokale Gerechtigkeit erfordert, dass Güter, die zentrale Aspekte des menschlichen Lebens betreffen, nach fairen Gesichtspunkten verteilt werden. Häufig gibt es für die Verteilung solcher Güter eigens dafür vorgesehene Institutionen, etwa Schulbehörden, zentrale Vergabestellen für Studienplätze und so fort.

Bei der Terminvergabe für Ämter und Impfzentren werden häufig Onlinesysteme eingesetzt, die nach dem Prinzip

(C) Der/die Autor:in(nen) 2021. Open Access: Dieser Artikel wird unter der Creative Commons Namensnennung 4.0 International Lizenz veröffentlicht (creativecommons.org/licenses/by/4.0/deed.de).

Open Access wird durch die ZBW - Leibniz-Informationszentrum Wirtschaft gefördert. funktionieren: „Wer zuerst kommt, mahlt zuerst.“ Diese urdemokratische Regel, die schon bei den Getreidemühlen Anwendung fand, gilt auch für Termine bei Konsulaten, Bürgerämtern etc. Solche Online-Buchungssysteme, die nach dem Windhundprinzip funktionieren, sind anfällig für Schwarzmärkte für Termine. Woran liegt das? Bei Onlinesystemen bekommt diejenige Person einen Termin, die sich zuerst einträgt. D.h., dass Geschwindigkeit zählt. Genau das können Firmen ausnutzen, die Termine mit sogenannten bots buchen, also Software nutzen, die die freien Termine in Sekundenschnelle findet und sofort reserviert. Oft kündigen die Firmen die Termine später wieder und buchen sie unter den Namen ihrer Klienten neu. Das ist wichtig, weil die Identität des Visumantragstellenden oder des Impflings während des Termins überprüft wird. Das bedeutet, dass der Termin nur von der Person wahrgenommen werden kann, deren Name im Buchungssystem gespeichert ist. Allerdings können Onlinesysteme normalerweise fiktive Namen nicht erkennen, was den Händlern ihre Arbeit erleichtert.

Der Handel mit Terminen ist weltweit zu beobachten - immer dann, wenn Online-Buchungssysteme genutzt werden, die nach dem Windhundprinzip funktionieren. Termine für Visa bei deutschen Konsulaten etwa in Beirut, Shanghai, Bischkek und Teheran gibt es nur gegen Geld. Dasselbe gilt auch für einige US-amerikanische und österreichische Konsulate. Auch Termine bei den Präfekturen in Frankreich sind mancherorts nur gegen hohe Geldsummen erhältlich. In Irland wurden Termine bei Einwanderungsbehörden von Firmen gebucht und weiterverkauft. Beim US-amerikanischen Department of Motor Vehicles (DMV), wo man seinen Führerschein verlängern kann, lassen sich gegen entsprechende Geldzahlungen rasch Termine finden, während diejenigen, die selbst einen Termin buchen möchten, monatelang warten müssen.

Ist das ein Problem? Der Handel mit Terminen kann bedeuten, dass die Terminsuchenden mit der höchsten Wertschätzung erfolgreich sind, da sie am meisten zu zahlen bereit sind. Das kann ein Vorteil sein. Allerdings wird es für viele öffentliche Dienstleistungen nicht als legitim angesehen, dass Geld da-

Prof. Dr. Dorothea Kübler ist Direktorin am Wissenschaftszentrum Berlin für Sozialforschung und Professorin an der Technischen Universität Berlin. 
rüber entscheidet, wer sie erhält. Denn es soll ja der gleiche Zugang für alle gelten, Arme und Reiche. Außerdem erscheint es nicht gerechtfertigt, dass die Firmen Gewinne machen mit Gütern und Dienstleistungen, die andere herstellen bzw. erbringen. Und schließlich zeigt sich, dass der Handel mit Terminen zu Verschwendung führt. Das liegt daran, dass Termine häufig unter falschen Namen gebucht werden, aber dann nicht verkauft werden, etwa weil der Preis zu hoch ist.

Die Forschungsfrage lautet also, wie sich der Handel mit Terminen verhindern lässt. Das Ziel muss sein, dass die Händler keinen Vorteil haben aufgrund der Geschwindigkeit. Das lässt sich dadurch erreichen, dass Termine nicht sofort vergeben werden, sondern dass sie nur in regelmäßigen Zeitabständen (z. B. täglich) zugeteilt werden. Das kann beispielsweise so aussehen, dass morgens neue Termine angeboten werden. Die Terminsuchenden haben dann bis abends Zeit, ihren Namen einzutragen, wenn sie Interesse an einem der Termine haben. Abends werden die Termine zugeteilt. Entscheidend ist dabei nicht, wer sich zuerst eingetragen hat. Falls es mehr Terminsuchende als Termine gibt, entscheidet der Zufall, wer einen Termin bekommt und wer nicht.

Es lässt sich zeigen, dass die Händler keine Gewinne mehr machen mit einem solchen Buchungssystem (Hakimov et al., 2021). Es ist leicht zu sehen, dass die Geschwindigkeit des Buchens keinen Vorteil mehr bedeutet. Jeder und jede Terminsuchende hat dieselbe Chance auf einen Termin wie die Firma, die mit Terminen handelt. Die Hauptforschungsergebnisse lassen sich in Kürze wie folgt zusammenfassen: Mit Hilfe eines spieltheoretischen Modells lässt sich zeigen, dass der Handel mit Terminen im Gleichgewicht beim vorgeschlagenen System unprofitabel ist, anders als beim bestehenden System, das nach dem Windhundprinzip funktioniert. In Laborexperimenten überprüfen wir den theoretisch vorhergesagten Zusammenhang zwischen dem Buchungssystem und der Profitabilität des Handels mit Terminen. Es zeigt sich, dass Händler in der Tat im neuen System kaum aktiv sind, während sie im bestehenden System praktisch alle Termine buchen und weiterverkaufen.

\section{Zwei Buchungssysteme im Vergleich: Modell und Experiment}

Wie sehen die Buchungssysteme nun genau aus? Das bestehende System, das Geschwindigkeit belohnt, bedeutet, dass die Händlerin zunächst alle Termine bucht, die sie wünscht, entweder mit den Namen ihrer Klienten oder mit fiktiven Namen. Die Terminsuchenden, die langsamer sind, buchen die verbleibenden Termine. Im vorgeschlagenen System bewerben sich dagegen die Händlerin und die Terminsuchenden um die angebotenen Termine in einem bestimmten Zeitraum. Wir nehmen an, dass die Händlerin viele Bewerbungen abgeben kann, aber die Terminsuchenden nur jeweils eine Bewerbung unter ihrem eigenen Namen. Eine Lotterie entscheidet dann darüber, wer einen Termin bekommt, falls es mehr Anfragen als Termine gibt. Falls nach der Zuteilung Termine storniert werden, gehen sie in zukünftigen Zuteilungen nach demselben Verfahren ein.

Das vorgeschlagene Zuteilungssystem hat zwei wichtige Eigenschaften: Es eliminiert erstens den Vorteil der Händler aufgrund der Geschwindigkeit und verhindert zweitens die Umgehung der Identifikation der Terminsuchenden. Beides ist nötig, um den Handel mit Terminen zu verhindern: Wenn die Händlerin schneller ist, kann sie mit Terminen handeln, auch wenn sie die Namen nach der Buchung nicht mehr verändern kann. In unserem Experiment ist genau das zu beobachten, da Stornierungen und Neubuchungen unter anderem Namen nicht möglich sind, der Handel mit Terminen aber trotzdem profitabel ist. Es gibt dafür auch Beispiele in der Praxis, wie etwa der Verkauf von Zugtickets in Indien, bei denen nur diejenigen ein Ticket erhalten, die über Firmen buchen, denen sie ihre Registrierungsdaten zur Verfügung stellen. Gleichzeitig gilt aber auch, dass eine Überprüfung der Identität erforderlich ist, selbst wenn das neue System übernommen wird, in dem die Geschwindigkeit des Buchens irrelevant ist. Wenn keine Überprüfung der Identität stattfindet, kann die Terminhändlerin im neuen System alle Termine buchen, indem sie sehr viele fiktive Bewerbungen abgibt, und sie dann später verkaufen. Das lässt sich bei Tickets für Sportereignisse und Konzerte beobachten, für die häufig Lotterien durchgeführt werden. Oft sind die Tickets sogar mit Namen versehen, aber beim Einlass in das Stadion wird die Identität nicht überprüft. Der Schwarzhandel für solche Tickets blüht infolgedessen.

Das spieltheoretische Modell lässt sich direkt im Experiment überprüfen. Sechs Teilnehmer bilden eine Gruppe: eine Firma (Händlerin) und fünf Terminsuchende. Die Teilnehmerin in der Rolle der Händlerin entscheidet, ob sie aktiv ist, und die Terminsuchenden entscheiden, ob sie einen Termin von der Händlerin kaufen oder sich selbst um einen Termin bemühen. Die Teilnehmer in der Rolle von Terminsuchenden erhalten eine Auszahlung, wenn sie einen Termin bekommen. Die Teilnehmer in der Rolle der Händler erhalten ihren Gewinn ausbezahlt.

Im Experiment haben wir die beiden Buchungssysteme implementiert, wobei „Immediate“ für das existierende und „Batch“ für das vorgeschlagene System stehen, jeweils mit hoher oder niedriger Nachfrage nach Terminen. Die theoretische Vorhersage für diese Märkte besagt, dass Firmen nur im bestehenden Buchungssystem mit hoher Nachfrage Gewinne machen, während sie im bestehenden System mit niedriger Nachfrage genau Nullgewinne machen, also indifferent zwischen Aktivität und Inaktivität sind. Im neuen Batch-Buchungssystem macht die Händlerin dagegen im Gleichgewicht Verluste. 
Abbildung 1

Aktivität der Terminhändlerin je nach Vergabesystem und Nachfrage nach Terminen

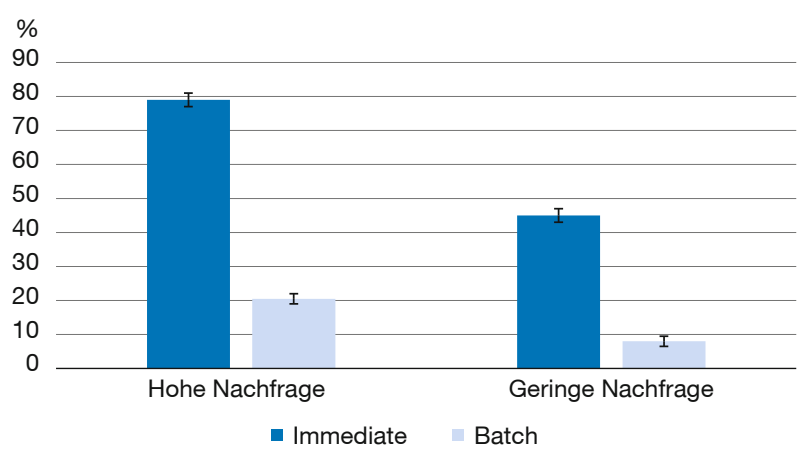

Quelle: Hakimov et al. (2021).

Die Abbildung 1 zeigt das Hauptergebnis des Experiments. Während die Händler im bestehenden Buchungssystem in etwa $80 \%$ aller Fälle aktiv sind, liegt der Anteil der aktiven Händler im neuen Batch-System nur bei $10 \%$ bis $20 \%$ je nach Höhe der Nachfrage. Wenn man betrachtet, welchen Nutzen die Terminsuchenden haben in Abhängigkeit vom Buchungssystem, dann zeigt sich, dass sie sowohl bei hoher als auch bei niedriger Nachfrage im neuen Batch-System immer besser dastehen als im herkömmlichen (Immediate) System, vgl. Abbildung 2.

Zusammenfassend zeigen wir, dass es im neuen BatchSystem für die Händlerin nicht profitabel ist, Termine zu verkaufen. Infolgedessen findet nur zu einem geringen Ausmaß Handel mit Terminen statt, und viele Händler treten gar nicht erst in den Markt ein. Die Terminsuchenden müssen infolgedessen im neuen System nichts für die Termine bezahlen. Marktdesign kann also dazu verwendet werden, Schwarzmärkte zu verhindern. Um es mit den Worten des Sozialphilosophen Michael Walzer auszudrücken, kann eine gute Ausgestaltung der Vergabe von Terminen für Impfungen und Visa sowie anderen öffentlich bereitgestellten Gütern verhindern, dass Geld eine Rolle spielt, „that money seeps in" (Walzer, 1983).

\section{Märkte ohne Geld: Schlussbemerkungen}

Was bedeutet das nun für die Vergabe von Impfterminen? Lassen sich die Erkenntnisse der Untersuchung für die Praxis fruchtbar machen? Es ist nicht schwer zu sehen, dass die Buchung eines Impftermins der Jagd nach Terminen bei Konsulaten, Tickets für Wimbledon, die Olympischen Spiele oder die Berliner Philharmoniker ähnelt. Es gab in Deutschland bei der Verteilung von Impfstoff erste Anzeichen von Schwarzmärkten, besonders zu Beginn der Impfkampagne. Aufgrund der Einbeziehung der Hausarztpraxen, die dezentral ihre Termine vergeben, aufgrund der strikten Priorisie-

\section{Abbildung 2}

Auszahlung der Terminsuchenden je nach Vergabesystem und Nachfrage nach Terminen

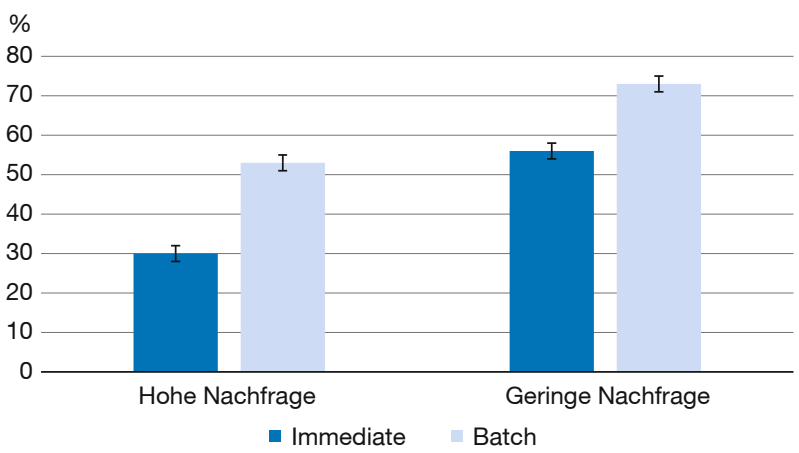

Quelle: Hakimov et al. (2021).

rung und der steigenden Menge an Impfstoff, waren illegale Terminvermittlungen aber wohl eher selten. Ein größeres Problem stellten zusammenbrechende Webseiten und stundenlange Warteschleifen bei der telefonischen Buchung dar. Beides sind logische Folgen des Buchungssystems, bei der die Geschwindigkeit entscheidet, wer einen Termin bekommt. Dieses Vergabesystem hat dazu geführt, dass ältere Menschen mit Kindern oder Enkeln, die ihnen helfen konnten, schneller zu einem Impftermin gekommen sind. Abgesehen davon gab es enorme Effizienzverluste durch zeitraubende Buchungsprozeduren. Wir schlagen deswegen vor, bei zukünftigen Verteilungsaufgaben das Windhundprinzip durch eine Lotterie zu ersetzen, falls es eine Übernachfrage gibt. Das stellt ex ante Fairness her, vereinfacht das Buchungsprozedere und verhindert Schwarzmärkte.

Für die Verteilung vieler wichtiger Güter spielt Geld keine entscheidende Rolle. Da viele dieser Güter aber knapp sind, braucht es Verteilungsmechanismen, die die Zuteilung organisieren nach Kriterien, die als angemessen angesehen werden. Die Grundlagen für die Erforschung von Zuteilungsverfahren verdanken wir David Gale, Lloyd Shapley, Al Roth und vielen anderen. Aufbauend auf ihren Arbeiten helfen Experimente dabei, besser zu verstehen, welche der vorgeschlagenen Mechanismen für die Teilnehmer akzeptabel sind und welche gut funktionieren (Roth, 2008). Während es gut erprobte Zuteilungsverfahren gibt, die in unterschiedlichen Varianten überall auf der Welt in verschiedenen Bereichen verwendet werden, gilt auch, dass jedes Verteilungsproblem anders ist. Neben der allgemeinen Theorie braucht es eine besondere Aufmerksamkeit für die Details, für den Kontext, damit das neue Marktdesign wirklich die Anforderungen erfüllt. Ein öffentliches Bewusstsein dafür zu schaffen, dass Vergabeverfahren nicht Schicksal sind, sondern besser oder schlechter gestaltet sein können, ist wichtig. Auch für so profane Dinge wie Buchungssysteme gilt, dass die Wissenschaft einen Beitrag leisten kann. 


\section{Literatur}

Elster, J. (1992), Local Justice. How Institutions Allocate Scarce Goods and Necessary Burdens, Russell Sage Foundation.

Hakimov, R., C.-P. Heller, D. Kübler und M. Kurino (2021), How to Avoid Black Markets for Appointments with Online Booking Systems, American Economic Review, 111(7), 2127-2151.
Roth, A. (2008). What Have We Learned From Market Design? Economic Journal, 118 (527), 285-310.

Walzer, M. (1983), Spheres Of Justice: A Defense Of Pluralism And Equality, Basic Books. 